\title{
Laser-induced forces between carbon nanotubes
}

\author{
David L. Andrews and David S. Bradshaw \\ School of Chemical Sciences, University of East Anglia, Norwich NR4 7TJ, UK
}

Received October 18, 2004

\begin{abstract}
Carbon nanotubes are the focus of intense research interest because of their unique properties and applications potential. We present a study based on quantum electrodynamics concerning the optical force between a pair of nanotubes under laser irradiance. To identify separate effects associated with the pair orientation and laser beam geometry, two different systems are analyzed. For each, an analytical expression for the laser-induced optical force is determined, and the corresponding magnitude is estimated. (C) 2005 Optical Society of America

OCIS codes: $020.5580,140.7010,220.4880,260.2110,270.5580,290.5890$.
\end{abstract}

Carbon nanotubes are currently the subject of intense research because of their unique nanostructures and remarkable combination of conductive, steric, and mechanical characteristics. ${ }^{1}$ Recent studies ${ }^{2,3}$ have shown that nanotubes can be optically trapped and manipulated by optical tweezers, ${ }^{4}$ exploiting the fact that the radiation pressure of inhomogeneous optical fields produces forces on neutral particles. ${ }^{5}$ In a separate development, other work ${ }^{6}$ has verified that significant optomechanical forces can be induced between particles through the application of an optical field, and differing methods of analysis have been utilized to derive this force with classical theory. ${ }^{7,8}$ In this Letter we use a quantum electrodynamics (QED) approach to determine the general result for optically induced forces between molecules, representing a form of stimulated scattering that may afford a new means for optical nanomanipulation. Two single-walled carbon nanotube (SWCNT) systems are analyzed in detail, differing in the angular disposition of the nanotubes and the incoming laser light: (i) parallel nanotubes disposed at a variable angle to the electric field vector of the incident light and (ii) nanotubes with variable mutual orientation averaged with respect to the field vector.

The laser-induced force $\mathbf{F}_{\text {ind }}$ between chemically identical nanotubes A and $\mathrm{B}$ can be determined from the optically induced energy shift $\Delta E_{\text {ind }}$. This energy shift has physical grounds similar to the well-known dispersion energy - an attractive interaction usually associated with the inductive coupling of fluctuating electric dipoles and that, in terms of molecular QED, involves the creation and annihilation of two virtual photons, ${ }^{9}$ i.e., four matter-photon interactions. In contrast, the laser-induced interaction reflects a process involving the absorption of a real input photon at one component and the stimulated emission of a real photon at the other, with one virtual photon acting as a messenger between them (Fig. 1). The throughput radiation suffers no overall change in state. As with the dispersion energy, the involvement of four matter-photon interactions requires the application of fourth-order perturbation theory:

$$
\Delta E_{\text {ind }}=\operatorname{Re}\left[\sum_{t, s, r} \frac{\left\langle i\left|H_{\text {int }}\right| t\right\rangle\left\langle t\left|H_{\text {int }}\right| s\right\rangle\left\langle s\left|H_{\text {int }}\right| r\right\rangle\left\langle r\left|H_{\text {int }}\right| i\right\rangle}{\left(E_{i}-E_{t}\right)\left(E_{i}-E_{s}\right)\left(E_{i}-E_{r}\right)}\right] .
$$

Here all Dirac brackets relate to states of the system comprising both nanotubes and the radiation field; $|i\rangle$ is the unperturbed state in which both molecules are in their electronic ground state; $|r\rangle,|s\rangle$, and $|t\rangle$ are virtual states; $E_{n}$ is the energy of state $|n\rangle$; and $H_{\text {int }}$ is the electric dipole interaction Hamiltonian. Considering real photon absorption at A with stimulated emission at $B$ and vice versa, accommodating virtual photon propagation between the two, allows us to determine the following concise result from Eq. (1):

$\Delta E_{\text {ind }}=\operatorname{Re}\left[\left(\frac{n \hbar c k}{\varepsilon_{0} V}\right) e_{i}^{(1)} \alpha_{i j}^{A} V_{j k}(k, \mathbf{R}) \alpha_{k l}^{B} e_{l}^{(2)} \exp (i \mathbf{k} \cdot \mathbf{R})\right]$,

Here the implied summation convention for repeated Cartesian tensor indices is denoted by subscripts, and $n, \mathbf{k}, \mathbf{e}, \hbar c k$, and $V$ denote the number of photons, their wave vector and polarization vector (assumed linear), the photon energy, and the quantization volume, respectively. Also $\alpha_{i j}$ are components of the molecular polarizability, and $V_{j k}$ are components of the retarded resonance electric dipole-electric dipole interaction tensor, which depends on intermolecular displacement vector $\mathbf{R}=\overrightarrow{\mathrm{AB}}$. Details of the perturbation method used here are given in earlier work. ${ }^{10}$

The first system to be examined in detail is a pair of SWCNTs aligned in parallel along the $X$ axis of a system frame, both perpendicular to $\mathbf{R}$, which is identified with the $Z$ axis, and with polarized throughput radiation having its e vector defined by the angles $\phi$ and $\theta$ with $\mathbf{R}$ in the $X Z$ plane and with

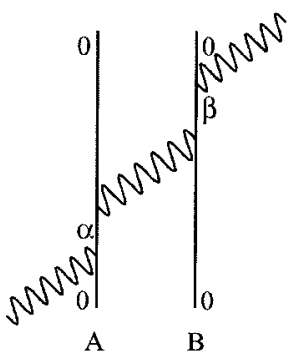

(a)

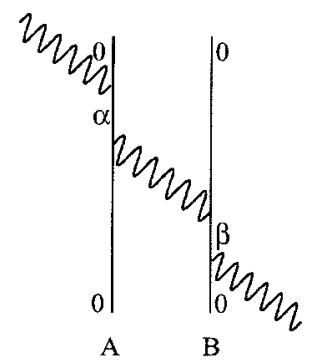

(b)
Fig. 1. Feynman diagrams (each with 23 further permutations) for calculation of the laser-induced interaction energy: 0 denotes the ground state, and $\alpha$ and $\beta$ are excited states for nanotubes A and B, respectively. 
the nanotube axis in the $X Y$ plane, respectively (Fig. 2 ). When we define the whole system in terms of this Cartesian frame, employ the explicit form of the $V_{j k}$ tensor, ${ }^{11}$ and recognize that for each nanotube $\alpha_{Y Y}$ $=\alpha_{Z Z}=\alpha_{\perp}$ and $\alpha_{X X}=\alpha_{\|}$, Eq. (2) becomes

$$
\begin{aligned}
\Delta E_{\text {ind }}= & \left(\frac{I}{4 \pi \varepsilon_{0}^{2} c}\right)\left[\left(\alpha_{\perp}^{2} \sin ^{2} \phi \sin ^{2} \theta+\alpha_{\|}^{2} \cos ^{2} \phi\right)\right. \\
& \times\left(\frac{\cos k R}{R^{3}}+\frac{k \sin k R}{R^{2}}-\frac{k^{2} \cos k R}{R}\right)-2 \alpha_{\perp}^{2} \\
& \left.\times \sin ^{2} \phi \cos ^{2} \theta\left(\frac{\cos k R}{R^{3}}+\frac{k \sin k R}{R^{2}}\right)\right] \cos (\mathbf{k} \cdot \mathbf{R}),
\end{aligned}
$$

where $I=n \hbar c^{2} k / V$ is the input irradiance and superscripts A and B are suppressed because of the chemical equivalence of the nanotubes. The laser-induced force $\mathbf{F}_{\text {ind }}=-\partial \Delta E_{\text {ind }} / \partial \mathbf{R}$ emerges as

$$
\begin{aligned}
F_{z}= & \left(\frac{1}{4 \pi \varepsilon_{0}^{2} c R^{4}}\right)\left(\left[\alpha_{\perp}^{2} \sin ^{2} \phi\left(1-3 \cos ^{2} \theta\right)+\alpha_{\|}^{2} \cos ^{2} \phi\right]\right. \\
& \times\left\{3 \hat{R}_{z} \cos k R \cos (\mathbf{k} \cdot \mathbf{R})+k R\left[3 \hat{R}_{z} \sin k R \cos (\mathbf{k} \cdot \mathbf{R})\right.\right. \\
& \left.+\hat{k}_{z} \cos k R \sin (\mathbf{k} \cdot \mathbf{R})\right]-k^{2} R^{2}\left[\hat{R}_{z} \cos k R \cos (\mathbf{k} \cdot \mathbf{R})\right. \\
& \left.\left.-\hat{k}_{z} \sin k R \sin (\mathbf{k} \cdot \mathbf{R})\right]\right\}-\left(\alpha_{\perp}^{2} \sin ^{2} \phi \sin ^{2} \theta\right. \\
& \left.+\alpha_{\|}^{2} \cos ^{2} \phi\right)\left\{k^{2} R^{2} \hat{R}_{z} \cos k R \cos (\mathbf{k} \cdot \mathbf{R})\right. \\
& +k^{3} R^{3}\left[\hat{R}_{z} \sin k R \cos (\mathbf{k} \cdot \mathbf{R})\right. \\
& \left.\left.\left.+\hat{k}_{z} \cos k R \sin (\mathbf{k} \cdot \mathbf{R})\right]\right\}\right) .
\end{aligned}
$$

The leading term in Eq. (4), $F_{z}^{0}$, in the short-range region $(k R \ll 1)$, is found by taking the leading terms in the Taylor series expansions of $\sin k R, \cos k R$, $\sin (\mathbf{k} \cdot \mathbf{R})$, and $\cos (\mathbf{k} \cdot \mathbf{R})$ to yield

$$
F_{z}^{0}=\left(\frac{3 I \hat{R}_{z}}{4 \pi \varepsilon_{0}^{2} c R^{4}}\right)\left[\alpha_{\perp}^{2} \sin ^{2} \phi\left(1-3 \cos ^{2} \theta\right)+\alpha_{\|}^{2} \cos ^{2} \phi\right] .
$$

On the assumption that the $\alpha_{\perp}$ and $\alpha_{\|}$values are consistent with the static polarizabilities ${ }^{12}$ for $R=2 \mathrm{~nm}$ and $I=1 \times 10^{16} \mathrm{~W} \mathrm{~m}^{-2}$, then for SWCNTs $200 \mathrm{~nm}$ in length and $0.4 \mathrm{~nm}$ in radius, Eq. (5) yields a force $F_{z}^{0}$

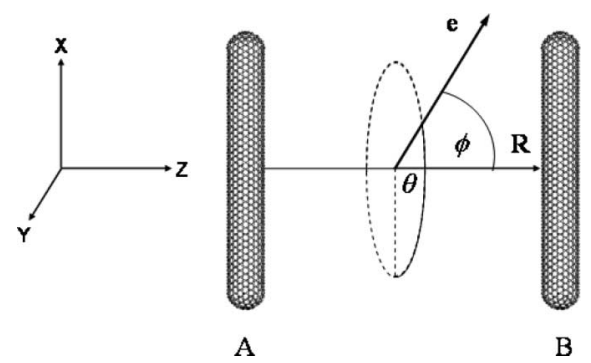

Fig. 2. Geometry of a parallel nanotube system irradiated in a fixed direction.

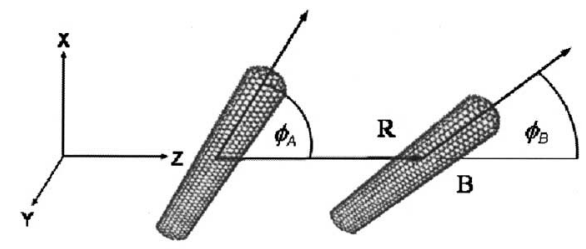

(a)

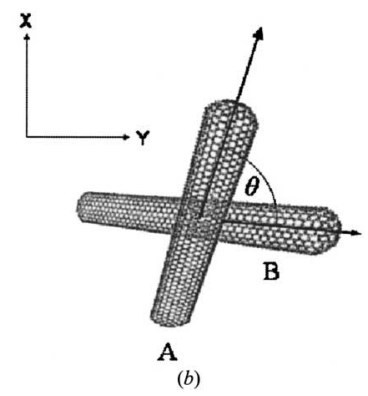

Fig. 3. Geometry of a nanotube pair with fixed orientation.

of (i) $\sim 10 \mu \mathrm{N}$ for $\phi=0^{\circ}$ (independent of $\theta$ ), (ii) $\sim$ $-1 \mathrm{pN}$ for $\phi=90^{\circ}$ and $\theta=0^{\circ}$, and (iii) $\sim 0.5 \mathrm{pN}$ for $\phi$ $=90^{\circ}$ and $\theta=90^{\circ}$.

In the second case the SWCNT pair has arbitrary mutual orientation but freely tumbles in the field of the input radiation. Here the angle between the long axis of each nanotube $\xi$ and the $\mathbf{R}$ vector (the $Z$ direction of the system frame) is defined as $\phi_{\xi}$ [Fig. $3(\mathrm{a})$ ]. Furthermore, $\theta$ is the angle between the longaxis projections of both nanotubes on the plane perpendicular to $\mathbf{R}$, and the $X$ direction of the system frame is chosen such that the long axis of nanotube $\mathrm{A}$ resides in the $X Z$ plane [Fig. 3(b)]. The nanotubes are isotropically averaged with respect to the light, accommodating the phase $\exp (i \mathbf{k R})$ in Eq. (2) reflects the creation and annihilation of a real photon at different positions, i.e., one at $\mathrm{A}$ and the other at $\mathrm{B}$. Hence by a phased average method ${ }^{13}$ we first obtain

$$
\begin{aligned}
\Delta E_{\text {ind }}= & \left(\frac{I}{\varepsilon_{0} c}\right) \operatorname{Re}\left[\frac{1}{3} j_{0}(k R) \alpha_{i j}^{A} V_{j k} \alpha_{k i}^{B}-\frac{3}{2} j_{2}(k R)\right. \\
& \left.\times\left(-\frac{1}{3} \hat{R}_{i} \alpha_{i j}^{A} V_{j k} \alpha_{k l}^{B} \hat{R}_{l}+\frac{1}{9} \alpha_{i j}^{A} V_{j k} \alpha_{k i}^{B}\right)\right] .
\end{aligned}
$$

Here $j_{n}(k R)$ are spherical Bessel functions defined by

$$
\begin{aligned}
& j_{0}(k R)=\frac{\sin k R}{k R} \\
& j_{2}(k R)=\left(\frac{-1}{k R}+\frac{3}{k^{3} R^{3}}\right) \sin k R-\frac{3 \cos k R}{k^{2} R^{2}} .
\end{aligned}
$$

Next, $\alpha_{i j}^{A}$ and $\alpha_{k i}^{B}$ are contracted with the $V_{j k}$ tensor and cast in terms of $\alpha_{\perp}$ and $\alpha_{\|}$. Finally, the laserinduced force is determined, yielding the following result: 


$$
\begin{aligned}
& F_{z}=\left(\frac{I k^{3} \hat{R}_{z}}{8 \pi \varepsilon_{0}^{2} c}\right)\left[\frac { \operatorname { c o s } ^ { 2 } k R - \operatorname { s i n } ^ { 2 } k R } { k R ^ { 2 } } \left\{\left(1-\eta \cos ^{2} \phi_{A}\right)\left[\alpha_{\|}^{2} \cos ^{2} \theta\left(1-\eta \cos ^{2} \phi_{B}\right)+\alpha_{\|} \alpha_{\perp} \sin ^{2} \theta\right]+\alpha_{\|} \alpha_{\perp} \sin ^{2} \theta\left(1-\eta \cos ^{2} \phi_{B}\right)\right.\right. \\
& \left.+\alpha_{\perp}^{2} \cos ^{2} \theta\right\}-\frac{\sin k R \cos k R}{k^{2} R^{3}}\left(6 \left\{\left(1-\eta \cos ^{2} \phi_{A}\right)\left[\alpha_{\|}^{2} \cos ^{2} \theta\left(1-\eta \cos ^{2} \phi_{B}\right)+\alpha_{\|} \alpha_{\perp} \sin ^{2} \theta\right]+\alpha_{\|} \alpha_{\perp} \sin ^{2} \theta\right.\right. \\
& \left.\left.\times\left(1-\eta \cos ^{2} \phi_{B}\right)+\alpha_{\perp}^{2} \cos ^{2} \theta\right\}-8 \alpha_{\|}^{2} \eta^{2} \sin \phi_{A} \sin \phi_{B} \cos \phi_{A} \cos \phi_{B} \cos \theta\right)+\frac{\sin ^{2} k R-\cos ^{2} k R}{k^{3} R^{4}} \\
& \times\left(6\left\{\left(1-\eta \cos ^{2} \phi_{A}\right)\left[\alpha_{\|}^{2} \cos ^{2} \theta\left(1-\eta \cos ^{2} \phi_{B}\right)+\alpha_{\|} \alpha_{\perp} \sin ^{2} \theta\right]+\alpha_{\|} \alpha_{\perp} \sin ^{2} \theta\left(1-\eta \cos ^{2} \phi_{B}\right)+\alpha_{\perp}^{2} \cos ^{2} \theta\right\}\right. \\
& \left.-14 \alpha_{\|}^{2} \eta^{2} \sin \phi_{A} \sin \phi_{B} \cos \phi_{A} \cos \phi_{B} \cos \theta+4 \alpha_{\|}^{2}\left(1-\eta \sin ^{2} \phi_{A}\right)\left(1-\eta \sin ^{2} \phi_{B}\right)\right)+\frac{\sin k R \cos k R}{k^{4} R^{5}} \\
& \times\left(16\left\{\left(1-\eta \cos ^{2} \phi_{A}\right)\left[\alpha_{\|}^{2} \cos ^{2} \theta\left(1-\eta \cos ^{2} \phi_{B}\right)+\alpha_{\|} \alpha_{\perp} \sin ^{2} \theta\right]+\alpha_{\|} \alpha_{\perp} \sin ^{2} \theta\left(1-\eta \cos ^{2} \phi_{B}\right)+\alpha_{\perp}^{2} \cos ^{2} \theta\right\}\right. \\
& \left.-48 \alpha_{\|}^{2} \eta^{2} \sin \phi_{A} \sin \phi_{B} \cos \phi_{A} \cos \phi_{B} \cos \theta+32 \alpha_{\|}^{2}\left(1-\eta \sin ^{2} \phi_{A}\right)\left(1-\eta \sin ^{2} \phi_{B}\right)\right)+\left(\frac{\cos ^{2} k R-\sin ^{2} k R}{k^{5} R^{6}}\right. \\
& \left.-\frac{\sin k R \cos k R}{k^{6} R^{7}}\right)\left(6 \left\{\left(1-\eta \cos ^{2} \phi_{A}\right)\left[\alpha_{\|}^{2} \cos ^{2} \theta\left(1-\eta \cos ^{2} \phi_{B}\right)+\alpha_{\|} \alpha_{\perp} \sin ^{2} \theta\right]+\alpha_{\|} \alpha_{\perp} \sin ^{2} \theta\left(1-\eta \cos ^{2} \phi_{B}\right)\right.\right. \\
& \left.\left.\left.+\alpha_{\perp}^{2} \cos ^{2} \theta\right\}-24 \alpha_{\|}^{2} \eta^{2} \sin \phi_{A} \sin \phi_{B} \cos \phi_{A} \cos \phi_{B} \cos \theta+24 \alpha_{\|}^{2}\left(1-\eta \sin ^{2} \phi_{A}\right)\left(1-\eta \sin ^{2} \phi_{B}\right)\right)\right],
\end{aligned}
$$

where $\eta=\left(\alpha_{\|}-\alpha_{\perp}\right) / \alpha_{\|}$. We find $F_{z}^{0}$, the short-range asymptote of Eq. (8), taking leading terms in the Taylor series expansion of $\sin k R$ and $\cos k R$,

$$
\begin{aligned}
F_{z}^{0}= & \left(\frac{I \hat{R}_{z}}{8 \pi \varepsilon_{0}^{2} c R^{4}}\right)\left(4 \left\{( 1 - \eta \operatorname { c o s } ^ { 2 } \phi _ { A } ) \left[\alpha_{\|}^{2} \cos ^{2} \theta\right.\right.\right. \\
& \left.\times\left(1-\eta \cos ^{2} \phi_{B}\right)+\alpha_{\|} \alpha_{\perp} \sin ^{2} \theta\right]+\alpha_{\|} \alpha_{\perp} \sin ^{2} \theta \\
& \left.\times\left(1-\eta \cos ^{2} \phi_{B}\right)+\alpha_{\perp}^{2} \cos ^{2} \theta\right\} \\
& -10 \alpha_{\|}^{2} \eta^{2} \sin \phi_{A} \sin \phi_{B} \cos \phi_{A} \cos \phi_{B} \cos \theta+4 \alpha_{\|}^{2} \\
& \left.\times\left(1-\eta \sin ^{2} \phi_{A}\right)\left(1-\eta \sin ^{2} \phi_{B}\right)\right) .
\end{aligned}
$$

With the same specifications as earlier we find that if parallel nanotubes are (i) also parallel to $\mathbf{R}$, the force from Eq. (9) has a magnitude of $\sim 8 \mu \mathrm{N}$, independent of $\theta$, or (ii) orthogonal to $\mathbf{R}$, then at $\theta=0^{\circ}$ or $\theta=90^{\circ}$, Eq. (9) yields $\sim 8 \mu \mathrm{N}$ or $\sim 3 \mathrm{nN}$, respectively.

Through a process of stimulated scattering by the pair, it has been shown that nanotubes experience a radiation-induced force under intense laser input. Calculations based on molecular QED have led to analytical results for widely ranging conditions, also giving physical insight into the mechanism. The results demonstrate that laser-induced forces can be either positive or negative according to conditions; the term binding force ${ }^{7,8}$ is potentially misleading. Detailed results have been given for two physically distinct cases, with calculations based on static polarizabilities yielding indicative values. The analysis suggests that the laser control of optomechanical forces offers considerable potential for the manipulation of carbon nanotubes. We suggest that one possible method for determining the magnitude of the laser-induced force may involve principles adapted from atomic force microscopy - a technique already associated with carbon nanotubes.

The authors thank the Engineering and Physical Sciences Research Council for funding. D. L. Andrews's e-mail address is david.andrews @physics.org.

\section{References}

1. G. Cao, Nanostructures and Nanomaterials: Synthesis, Properties and Applications (Imperial College Press, London, 2004), p. 238.

2. J. Plewa, E. Tanner, D. M. Mueth, and D. G. Grier, Opt. Express 12, 1978 (2004), http:// www.opticsexpress.org.

3. S. Tan, H. A. Lopez, C. W. Cai, and Y. Zhang, Nano Lett. 4, 1415 (2004).

4. J. E. Molloy, K. Dholakia, and M. J. Padgett, J. Mod. Opt. 50, 1501 (2003).

5. A. Ashkin, Proc. Natl. Acad. Sci. U.S.A. 94, 4853 (1997).

6. M. M. Burns, J.-M. Fournier, and J. A. Golovchenko, Phys. Rev. Lett. 63, 1233 (1989).

7. F. Depasse and J.-M. Vigoureux, J. Phys. D 27, 914 (1994).

8. P. C. Chaumet and M. Nieto-Vesperinas, Phys. Rev. B 64, 035422 (2001).

9. D. P. Craig and T. Thirunamachandran, Molecular Quantum Electrodynamics (Dover, Mineola, N.Y., 1998), pp. 152-163.

10. P. Allcock, R. D. Jenkins, and D. L. Andrews, Phys. Rev. A 61, 023812 (2000).

11. G. J. Daniels, R. D. Jenkins, D. S. Bradshaw, and D. L. Andrews, J. Chem. Phys. 119, 2264 (2003).

12. G. Y. Guo, K. C. Chu, D.-S. Wang, and C.-G. Duan, Comput. Mater. Sci. 30, 269 (2004).

13. D. L. Andrews and M. J. Harlow, Phys. Rev. A 29, 2796 (1984). 